\title{
Using Bernoulli Equation to Solve Burger's Equation
}

\author{
Saad N. AL-Azawi* \\ Muna Saleh*
}

Received 20, December, 2012

Accepted 12, June, 2013

\begin{abstract}
:
In this paper we find the exact solution of Burger's equation after reducing it to Bernoulli equation. We compare this solution with that given by Kaya where he used Adomian decomposition method, the solution given by chakrone where he used the Variation iteration method (VIM)and the solution given by $\mathrm{Eq}(5)$ in the paper of $\mathrm{M}$. Javidi. We notice that our solution is better than their solutions.
\end{abstract}

Key words: Burger's equation, kdv equation, PDF.

\section{Introduction:}

We consider the following Burger's equation

$$
\mathrm{u}_{\mathrm{t}}+\mathrm{uu}_{\mathrm{x}}-\lambda \mathrm{u}_{\mathrm{xx}}=0
$$

Where $\lambda$ is positive parameter

This equation arises in various areas of science. Equation (1.1) has been used in the study of the propagation through liquid-filled elastic tube[1].and description for shallow water waves on a viscous fluid [2].Equation(1.1)is used as a model for traffic flow [3].Many Researchers had proposed various kinds of exact and numerical solution $[4,5,6,7,8,9,10]$ where they used Adomian decomposition method, variation iteration method, Galerkin method.

\section{Derivation of Burger's}

\section{equation.[9]}

We recall the differential form of the nonlinear conservation equation

$\frac{\partial \mathrm{p}}{\partial \mathrm{t}}+\frac{\partial \mathrm{q}}{\partial \mathrm{x}}=0$

To investigate the nature of the discontinuous solution or shock waves, we assume a function relation
$\mathrm{q}=\mathrm{Q}(\mathrm{p})$ and allow a jump discontinuity for $\mathrm{p}$ and $\mathrm{q}$.

In many physical problems of interest it would be a better approxinfdtibh to assume that $\mathrm{q}$ is a function of the density gradient $p_{x}$ as well as p.A simple model is to take

$\mathrm{q}=\mathrm{Q}(\mathrm{p})-\mathrm{vp}_{\mathrm{x}}$

Where $v$ is a positive constant .Substituting (2.2) into (2.1), we obtain the nonlinear diffusion equation

$$
\mathrm{p}_{\mathrm{t}}+\mathrm{c}(\mathrm{p}) \mathrm{p}_{\mathrm{x}}=v \mathrm{p}_{\mathrm{xx}}
$$

Where, $\mathrm{c}(\mathrm{p})=\mathrm{Q}^{\prime}(\mathrm{p})$

We multiply (2.3) by $\mathrm{c}^{\prime}(\mathrm{p})$ to obtain

$\mathrm{c}^{\prime}(\mathrm{p}) \mathrm{p}_{\mathrm{t}}+\mathrm{c}(\mathrm{p}) \mathrm{c}^{\prime}(\mathrm{p}) \mathrm{p}_{\mathrm{x}}=v c^{\prime}(\mathrm{p}) \mathrm{p}_{\mathrm{xx}}$

Hence:

$c_{\mathrm{t}}+\mathrm{cc}_{\mathrm{x}}=\mathrm{vc}^{\prime}(\mathrm{p}) \mathrm{p}_{\mathrm{xx}}$

And therefore:

$c_{\mathrm{t}}+c c_{\mathrm{z}}=v c^{\prime}(\mathrm{p}) \mathrm{p}_{\mathrm{xz}}=v c_{\mathrm{xz}}-c^{\prime \prime}(\mathrm{p}) \mathrm{p}_{\mathrm{z}}^{2}$

If $Q(p)$ is a quadratic (2.1)hetion in $\mathrm{p}$,then $\mathrm{c}(\mathrm{p})$ is linear in $\mathrm{p}$, $\operatorname{andc}^{s t}(\mathrm{p})=0$.

Consequently (2.4) becomes

\footnotetext{
* Department of Mathematics. College of science for women. Baghdad University
} 
$c_{\mathrm{t}}+\mathrm{cc}_{\mathrm{x}}=\mathrm{vc} \mathrm{c}_{\mathrm{xx}}$

As a simple model of turbulence, $c$ is replaced by the fluid velocity field $\mathrm{u}(\mathrm{x}, \mathrm{t})$ to obtain the well-known Burgers equation as

$\mathrm{u}_{\mathrm{t}}+\mathrm{uu}_{\mathrm{x}}=\mathrm{vu}_{\mathrm{xx}}$

Where $v$ is the kinematic viscosity.Thus, the Burgers equation is a balance between time evaluation, nonlinearity and diffusion.

\section{Solution of Burger's}

\section{equation.}

To solve Burger's equation (1.1), we can assume the

solution $\mathrm{u}(\mathrm{x}, \mathrm{t})=\mathrm{v}(\mathrm{z})$

Where

$\mathrm{z}=\mathrm{x}-\lambda \mathrm{t}$

$-v$ is called the travelling wave solution-Substituting (3.1) into (1.1) becomes

$v_{z z}=\left(\frac{v}{\lambda}-1\right) v_{z}$

Rewrite (3.2) as a system of first order O.D.E's

Let

$\mathrm{r}_{1}=\mathrm{v}$

$\mathrm{r}_{2}=\mathrm{v}_{\mathrm{z}}$

Where $r_{1}, r_{2}$ represent the velocity and a acceleration respectively.

Then

$\mathrm{r}_{1}^{\prime}=\mathrm{v}=\mathrm{r}_{2}$

$\mathrm{r}_{2}^{\prime}=\mathrm{v}_{\mathrm{zz}}=\left(\frac{\mathrm{r}_{1}}{\lambda}-1\right) \mathrm{r}_{2}$

This system has an infinite number of equilibrium points which is

$\mathrm{r}_{1}$ - axis

To solve (3.4) divide the two equations to get $\frac{\mathrm{dr}_{2}}{\mathrm{dr}_{1}}=\frac{\mathrm{r}_{1}}{\lambda}-1$

i.e.

$$
\mathrm{dr}_{2}=\left(\frac{\mathrm{r}_{1}}{\lambda}-1\right) \mathrm{dr}_{1}
$$

$\mathrm{r}_{2}=\frac{\mathrm{r}_{1}^{2}}{2 \lambda}-\mathrm{r}_{1}+\mathrm{c}$

Where $\mathrm{c}$ is an arbitrary constant.

Hence, from (3.3) we get

$v_{z}+v=\frac{v^{2}}{2 \lambda}+c$

Now, let

$\mathrm{w}=\mathrm{v}+\mathrm{k}$

[In order to eliminate $\mathrm{c}$ from equation (3.5)] substituting (3.6) into (3.5)

becomes

$w_{z}+w-k=\frac{(w-k)^{2}}{2 \lambda}+c$

i.e.

$w_{z}+\left(1+\frac{k}{\lambda}\right) w=\frac{w^{2}}{2 \lambda}+\frac{k^{2}}{2 \lambda}+k+c$

Let $\frac{\mathrm{k}^{2}}{2 \lambda}+\mathrm{k}+\mathrm{c}=0$ to get

$\mathrm{k}=-\lambda \mp \sqrt{\lambda^{2}-2 \lambda c}$

Then (3.7) becomes

$w_{z}+\left(1-\frac{k}{\lambda}\right) w=\frac{w^{2}}{2 \lambda}$

Notice that equation (3.9) represents Bernoulli equation. To solve it Suppose

$\eta=w^{-1} \rightarrow \eta_{z}=-w^{-2} w_{z}$

Substituting (3.10) int $(3.349)$

$w^{-2} w_{z}+\left(1+\frac{k}{\lambda}\right) w^{-1}=\frac{1}{2 \lambda}$

We get

$\eta_{z}-\left(1+\frac{k}{\lambda}\right) \eta=\frac{-1}{2 \lambda}$ 
This equation is first-order linear differential equation, its integrating factor is

$I(z)=e^{-\left(1+\frac{k}{h}\right)} z$

And its solution is gives by

$\eta I=\frac{1}{2(\lambda+\kappa)} e^{-\left(\frac{k}{h}+1\right) z}+\alpha$

Where $\alpha$ is an arbitrary constant so,

$\eta=\left(\frac{1}{2(\lambda+k)}+\alpha e^{\left(1+\frac{k}{\lambda}\right) z}\right.$

From (3.10), we get the solution of Bernoulli equation (3.9)

$$
w(z)=\frac{1}{\frac{1}{2(\lambda+\kappa)}+\alpha e^{\left(1+\frac{\mathrm{K}}{\lambda}\right) z}}
$$

By using (3.6) and (3.11)

Now, from (3.6) $v=w-k$ so the traveling wave solution (3.1) becomes

$$
u(x, t)=v(z)=w(z)-k
$$

$$
\mathrm{u}(\mathrm{x}, \mathrm{t})=\frac{1}{\frac{1}{2(\lambda+\kappa)}+\alpha \mathrm{e}^{\left(1+\frac{\mathrm{K}}{\mathrm{\gamma}}\right)(\mathrm{x}-\mathrm{h} \mathrm{t})}}-\mathrm{k}
$$

Where $\mathrm{k}$ is given in (3.8) and $\alpha$ is an arbitrary constant where $\mathrm{k}=-\lambda \mp \sqrt{\lambda^{2}-2 \lambda \mathrm{c}}$ And $\alpha, c$ are arbitrary constants.

\section{The solution of Burger's} equation with Dirichlet conditions.

From (3.12) the solution of Burger's equation is

$$
\mathrm{u}(\mathrm{x}, \mathrm{t})=\frac{1}{\frac{1}{2(\lambda+\kappa)}+\alpha \mathrm{e}^{\left(1+\frac{\mathrm{K}}{2}\right)(\mathrm{x}-\mathrm{\lambda} \mathrm{t})}}-\mathrm{k}
$$

Dirichlet conditions are
$\mathrm{BC} 1: \mathrm{u}(0, \mathrm{t})=\mathrm{g}(\mathrm{t})$

$0 \leq t \leq t_{1}$

$\mathrm{BC} 2: \mathrm{u}(1, \mathrm{t})=\mathrm{h}(\mathrm{t})$

Clearly u $(0, \quad 0) \quad=0 \quad$ (stationary case)

$\mathrm{BC} 1 \rightarrow$

$$
\mathrm{u}(\mathrm{x}, \mathrm{t})=\frac{1}{\frac{1}{2(\lambda+\kappa)}+\alpha \mathrm{e}^{-\left(1+\frac{\mathrm{K}}{2}\right) / \mathrm{t}}}-\mathrm{k}=\mathrm{g}(\mathrm{t})
$$

$\mathrm{BC} 2 \rightarrow$

$$
\mathrm{u}(\mathrm{x}, \mathrm{t})=\frac{1}{\frac{1}{2(\lambda+\kappa)}+\alpha \mathrm{e}^{\left(1+\frac{K}{2}\right)(1-\lambda \mathrm{t})}}-\mathrm{k}=\mathrm{h}(\mathrm{t})
$$

$\mathrm{u}(0,0)=0 \rightarrow$

$\frac{1}{\frac{1}{2(\lambda+\kappa)}+\alpha}-k=0$

Therefore,

$\alpha=\frac{1}{\mathrm{k}}-\frac{1}{2(\lambda+\mathrm{k})}=\frac{2 \lambda+\mathrm{k}}{2(\lambda+\mathrm{k}) \mathrm{k}}$

Remember that from (3.8)

$\mathrm{k}=-\lambda \mp \sqrt{\lambda^{2}-\lambda c}$

And from (4.2)

$h(0)=\frac{1}{\frac{1}{2(\lambda+\kappa)}+\alpha e^{\left(1+\frac{K}{2}\right)}}-k$

From which we get

$\alpha=\left[\frac{1}{h(0)+k}-\frac{1}{2(\lambda+k)}\right] e^{\frac{-(\lambda+k)}{\lambda}}$

From(4.3) and (4.4)we determine $\alpha$ and $c$

After determining these constants we 
find the solution of Burger's equation with Dirichlet conditions.

As a special case let $\lambda=2$ and $c=0.75$ then from (3.8) we get

$\mathrm{k}=-3$ or -1

And from (4.3) we get

$\alpha=\frac{1}{6}$ if $\mathrm{k}=-3$

Or

$\alpha=\frac{-3}{2}$ if $\mathrm{k}=-1$

\section{Case}

$\underline{1}: \lambda=2, c=0.75, k=-3, \alpha=\frac{1}{6}$

$\mathrm{u}(\mathrm{x}, \mathrm{t})=\frac{1}{\frac{-1}{2}+\frac{1}{6} \mathrm{e}^{-0.5(\mathrm{x}-2 \mathrm{t})}}+3$

\section{Case 2:}

$\lambda=2, \mathrm{c}=0.75, \mathrm{k}=-1, \alpha=\frac{-3}{2}$

$\mathrm{u}(\mathrm{x}, \mathrm{t})=\frac{1}{\frac{1}{2}-\frac{3}{2} \mathrm{e}^{0.5(\mathrm{x}-2 \mathrm{t})}}+1$

The graphs of (4.5),(4.6) are in figures (1)and(2) respectively.

The two shapes have the same qualitative behavior but quantitavely different.

\section{Comparison.}

- The solution of this equation obtained by Kaya

$\mathrm{u}(\mathrm{x}, \mathrm{t})=\frac{\left(\alpha+\beta+(\beta-\alpha) \mathrm{e}^{(\mathrm{Q})}\right)}{\left(1+\mathrm{e}^{(\mathrm{C})}\right)}$

Where $\zeta=\frac{a}{\psi}(\mathrm{x}-\beta \mathrm{t}-\eta)$ where is not easier than our solution.

- The solution obtained by Omar is approximated solution, while our solution is exact solution.

- The solution obtained by Javidi

$\mathrm{u}(\mathrm{x}, \mathrm{t})=\frac{\left(0.1 \mathrm{e}^{-\mathrm{A}}+0.5 \mathrm{e}^{-\mathrm{B}}+\mathrm{e}^{-\mathrm{C}}\right)}{\left(\mathrm{e}^{-\mathrm{A}}+\mathrm{e}^{-\mathrm{B}}+\mathrm{e}^{-\mathrm{C}}\right)}$

Where,

$\mathrm{A}=\frac{0.05}{\mu}(x-0.5+4.95 t)$,

$\mathrm{B}=\frac{0.05}{\mu}(x-0.5+0.75 t)$ and

$\mathrm{C}=\frac{0.05}{\mu}(\mathrm{x}-0.5+0.375 \mathrm{t})$

Clearly our solution is easier than his solution.

when

$$
, \mathrm{k}=0.2, \mathrm{c}=0 \quad \begin{aligned}
& \alpha=-1.72, \\
& (4.5)
\end{aligned} \quad \lambda=0.1
$$

in the solution function

$$
\mathrm{u}(\mathrm{x}, \mathrm{t})=\frac{1}{\frac{1}{2(\lambda+\kappa)}+\alpha \mathrm{e}^{\left(1+\frac{\mathrm{K}}{2}\right)(\mathrm{x}-\mathrm{\gamma} \mathrm{t})}}-\mathrm{k}
$$

where $\mathrm{t}=[0: 0.1: 0.4], \mathrm{x}=[-10: 5: 10]$

This figure classifies the wave at the beginning of the earth quake.

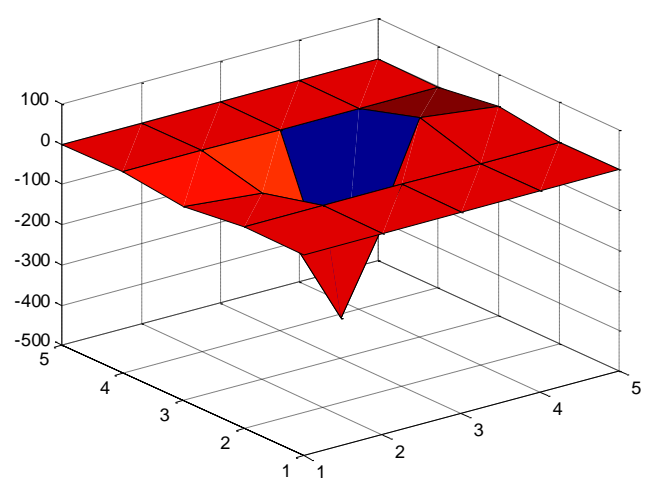




$$
\underline{\text { Case 1 }}: \lambda=2, c=0.75, k=-3, \alpha=\frac{1}{6}
$$

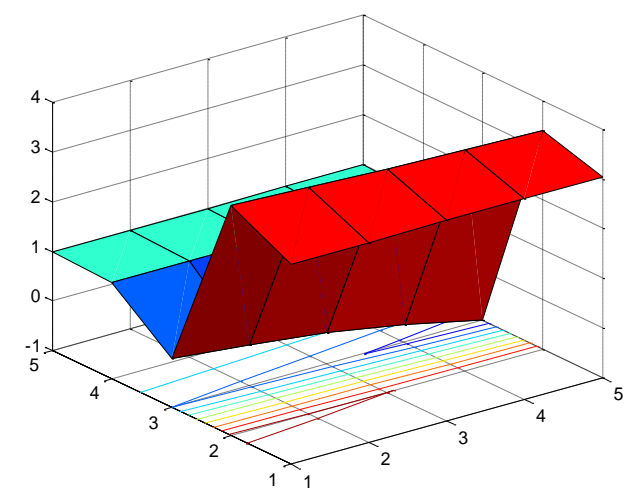

Fig.-1-

Case2: $\lambda=2, c=0.75, k=-1, \alpha=\frac{-3}{2}$

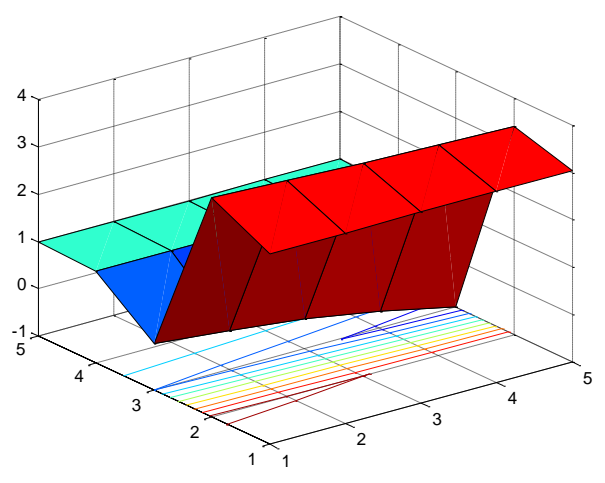

Fig.-2-

\section{References:}

1. R.S.Jonson, 1970.A Non Linear Equation Incorporating Damping and Disper sion.J.Phys.Mech .(42)49-60.

2. R.S.Jonson, 1972.Shallow Water Wave in Viscous Fluid-the Undular Bore.phys- Fluids (15) 1693-1699.
3. Md Abdur Rab, 2012.Some Travelling Wave Solutions of KdVBurgers Equation.Int. Journal of Math. Analysis, Vol. 6(22): 1053 - 1060

4. Dogan Kaya, 2004.An application of the decomposition method for the KdVB Equation .Applied Mathematics and Computation 15(2)279-288.

5. Omar Chakrone, Okacha Diyer, Driss Sbibih 2010.Improved numerical solution of

Burger's equation.Bol.Soc.Paran .Mat. (3s)v.28 (2):9-14

6. J.Biazar and H.H.Ghazvini, 2009. Exact and numerical for non linear Burger's Equation by VIM. Mathematical and Computer Modelling, 1394-1400.

7. J.H.He, 2007.Variational iteration method - Some recent results and new inter-Pretations. Mathematical Computer.

8. X.H.Zhang, J.Ouyang, L.Zhang, 2009.Element - free characteristic Galerkin method for Buger's equation. Engineering Analysis with Boundary Elements, 356-362.

9. Jawad Kadhim, 2007.Non-Classical Variation Formulation Approach for solving one-Dimensional Non linear Partial Differential Burger's Problem.M.S.C thesis, Baghdad University

10. M. Javidi, 2006.A numerical solution of Burgers equation based on modied extended BDF scheme. International Mathematical Forum, 1( 32) $1565-1570$

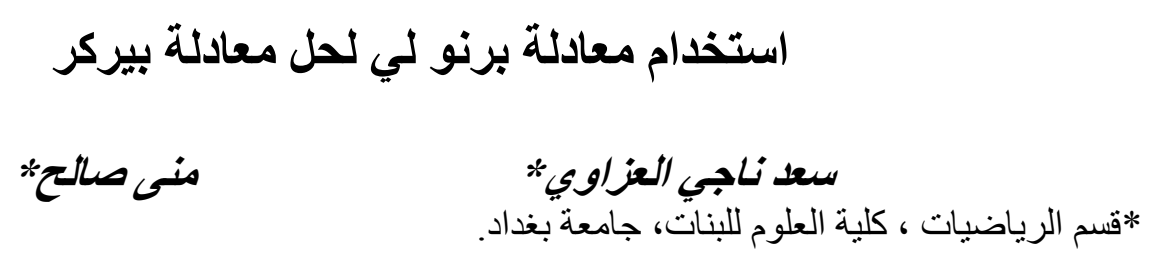

الخلاصة:

حصلنا من بحثنا هذا على الحل المضبوط (Exact) لمعادلة بيركر بعد تحويلها إلى معادلة برنو لي وتمت مقارنة التحنة

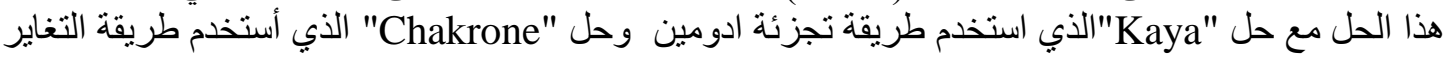
المتكرر وحل "Javidi" المعطى بالعلاقة (5)وتنبين ان حلنا هذا افضل من حلول لهم للبساطُه الصيغة 